\title{
Dipolar dark matter and CMB B-mode polarization
}

\author{
S. Mahmoudi ${ }^{1, \mathrm{a}}$, M. Haghighat ${ }^{1,2, \mathrm{~b}}$, S. Al. Modares Vamegh ${ }^{1, \mathrm{c}}$, R. Mohammadi ${ }^{3,4, \mathrm{~d}}$ (D) \\ ${ }^{1}$ Physics Department, College of Sciences, Shiraz University, 71454 Shiraz, Iran \\ 2 Islamic World Science Citation Center, 71946-94173 Shiraz, Iran \\ ${ }^{3}$ Iranian National Science and Technology Museum (INMOST), P.O. Box: 11369-14611, Tehran, Iran \\ ${ }^{4}$ School of Astronomy, Institute for Research in Fundamental Sciences (IPM), P.O. Box 19395-5531, Tehran, Iran
}

Received: 31 May 2018 / Accepted: 28 April 2020 / Published online: 11 May 2020

(C) The Author(s) 2020

\begin{abstract}
We consider dark matter particles as singlet fermionic particles carrying magnetic dipole moment to explore its contribution on the polarization of the cosmic microwave background (CMB) photons. We show that Dirac fermionic dark matter particles have no contribution on the CMB polarization. However, in the case of Majorana dark matter, this type of interaction leads to the B-mode polarization in the presence of primordial scalar perturbations which is in contrast with the standard scenario for the CMB polarization. We numerically calculate the B-mode power spectra and plot $C_{l}^{B B}$ for different dark matter masses and the $r$ parameter. We show that dark matter particles with masses less than $100 \mathrm{MeV}$ have a valuable contribution on $C_{l}^{B B}$. Meanwhile, dark matter particles with mass $\mathrm{m}_{\mathrm{DM}} \leq 50 \mathrm{MeV}$ for $r=0.07\left(\mathrm{~m}_{\mathrm{DM}} \leq 80 \mathrm{MeV}\right.$ for $\left.r=0.09\right)$ can be excluded experimentally. Furthermore, our results put a bound on the magnetic dipole moment about $\mathrm{M} \leq 10^{-16} e \mathrm{~cm}$ in agreement with the other reported constraints.
\end{abstract}

\section{Introduction}

The nature of dark matter (DM) and its interactions is one of the most important questions in cosmology and particle physics. Although there is some wealth of cosmological evidence for existing DM, from galactic clusters and velocity curves of spinning galaxies to gravitational lensing [1-8], its particle properties have remained elusive. To explore the nature of DM, different experiments have been proposed such as DAMA/LIBRA collaboration at Gran Sasso [9], CoGeNT collaboration at the Soudan Laboratory underground [10] and CDMS collab-

\footnotetext{
a e-mail: s.mahmoudi@shirazu.ac.ir

b e-mail: M.Haghighat@ shirazu.ac.ir

c e-mail: s.modares@shirazu.ac.ir

de-mail: rmohammadi@ipm.ir (corresponding author)
}

oration [11] which are introduced to detect DM directly. In these experiments, the scattering of DM from nucleons can be described by multiple interactions. In fact, DM particle has zero electric charge and therefore in the simplest extension of the standard model, it can be coupled to photon through an intrinsic electric and /or magnetic dipole moments which is well-known as dipolar DM (DDM) model [12-16]. However, the DDM model can successfully explain some claims of DAMA/LIBRA and COGENT collaborations $[17,18]$.

The CMB photons are expected to be linearly polarized due to the anisotropic Compton scattering around the epoch of recombination. Meanwhile, according to the standard scenario of cosmology, there is no physical mechanism to generate circularly polarized radiation at the last scattering surface. However, studies conducted in recent years show that the interaction between photons and matter can convert or generate the polarization states of photons in different situations. For instance, the linear polarization of the $\mathrm{CMB}$ photons can be converted to the circular one in the presence of background fields or due to the effects of particle scattering which has been widely discussed in the literature [19-30]. In this paper, we consider the DDM model with a singlet spin $\frac{1}{2}$ fermion as DM particles to examine the effects of magnetic dipole moments on the CMB photon polarization.

Generally, the CMB polarization pattern has two geometrical components, E-mode and B-mode. These modes based on the Stokes parameters $\mathrm{U}$ and $\mathrm{Q}$ can form an independent local coordinate system [31-35]. According to the standard model of cosmology, while E-mode polarization of the $\mathrm{CMB}$ can be produced by Compton scattering at the last scattering surface in the presence of scalar and tensor perturbations, its B-mode polarization pattern can only be produced at the presence of tensor perturbations. Nevertheless, it has been shown that it is also possible to produce B-mode 
polarization in the presence of scalar perturbations. Since the detection of the B-mode polarization can provide a unique tool to investigate the $\mathrm{CMB}$ perturbations, it is important to identify all potential sources of the B-mode polarization. As the new sources, for instance, in [36] the effect of the Faraday rotation due to the uniform magnetic field on the CMB is investigated and it is shown that a nonvanishing Bmode polarization can be produced through Faraday rotation. In [37,38], the authors have discussed that photon-neutrino interaction in the presence of scalar perturbations could be considered as one of the sources of the CMB B-mode polarization. It is also shown that the Compton scattering in the non-commutative space-time can generate the B-mode polarization of the CMB [39] and the possibility of the producing B-mode polarization pattern due to polarized Compton scattering in the presence of scalar perturbations has been discussed in [40].

However, the parameter which characterizes the amplitude of the metric tensor perturbation is $r=\mathrm{P}_{\mathrm{T}} / \mathrm{P}_{\mathrm{S}}$ where $P_{T}=A_{T}\left(k / k_{\circ}\right)^{n_{T}-1}$ and $P_{S}=A_{S}\left(k / k_{\circ}\right)^{n_{S}-1}$ are, respectively, the power spectra of tensor and scalar metric perturbations and $n_{T, S}$ and $A_{T, S}$ are their spectral indices and amplitudes. The $r$ parameter is usually calculated by comparing the B-mode and E-mode power spectra. Recent measurements of BICEP2 + Keck Array + Planck (BKP) report an upper bound $r_{0.002}<0.09$ [41].

In this work, we will show that the magnetic like component of the CMB polarization (B-mode polarization) can be produced by the photon-DM interaction in the presence of scalar perturbations. The paper is organized as follows: we introduce the effective Lagrangian for the interaction of DDM with photons in Sect. 2. Then we give a brief introduction to the Stokes parameters and drive the time evolution of these parameters in terms of the photon-DM scattering in Sect. 3. The power spectrum is evaluated numerically in Sect. 4. We compare our results with the experimental data and give some discussion in Sect. 5.

\section{Dipolar dark matter model}

A particle as a candidate for DM is generally known as a stable or relatively stable particle that does not interact electromagnetically. However, in recent years, there are some interests in the study of the electromagnetic interactions of DM. Such a particle has not probably the electric charge otherwise it has significant interaction with the photons and could be easily detected. But this particle can weakly couple with the electromagnetic field through loop corrections. The most general form for the electromagnetic current between fermions consistent with the Lorentz covariance and the Ward identity can be written as follows [42]:

$$
\begin{aligned}
J_{\mu}^{e m}= & \bar{\psi}\left(p^{\prime}\right)\left[\gamma_{\mu} F_{1}\left(q^{2}\right)-\gamma_{\lambda} \gamma_{5}\left(g_{\mu}^{\lambda} q^{2}-q^{\lambda} q_{\mu}\right) G_{1}\left(q^{2}\right)\right. \\
& \left.+\sigma_{\mu \nu} q^{\nu}\left[F_{2}\left(q^{2}\right)+\gamma_{5} G_{2}\left(q^{2}\right)\right]\right] \psi(p),
\end{aligned}
$$

where $F_{1}, G_{1}, F_{2}$ and $G_{2}$ are the electric charge, anapole, magnetic and electric dipole form factors, respectively. The current $J_{\mu}^{e m}$ can couple with photons through dipolar part as follows

$\mathcal{L}_{\mathrm{DDM}}=-\frac{i}{2} \bar{\psi} \sigma_{\mu \nu}\left(M+\gamma^{5} D\right) \psi F^{\mu \nu}$,

where $F^{\mu v}$ is the electromagnetic field, $M$ and $D$ are permanent magnetic and electric dipole moment, respectively and $\sigma^{\mu \nu}=\frac{i}{2}\left[\gamma^{\mu}, \gamma^{\nu}\right]$. It is necessary to mention that the permanent dipole moment can be defined just for Dirac particle and Majorana particle can not have a permanent dipole moment. However, in the case of Majorana fermions, it is possible to define transition moments between different mass eigenstates which their interactions with photons are described by $[13,43]$

$\mathcal{L}_{\mathrm{DDM}}=-\frac{i}{2} \bar{\psi}_{2} \sigma_{\mu \nu}\left(M_{12}+\gamma^{5} D_{12}\right) \psi_{1} F^{\mu \nu}+$ H.C.,

where $M_{12}$ and $D_{12}$ stand for the transition magnetic moment and electric moment, respectively. The Lagrangian (2) and (3) form the basis of the DDM model [12]. Therefore, the fermionic DM-particle can interact with photons via electric and magnetic dipole moments [44-46].

\section{Time evolution of stokes parameters due to DDM-photon scattering}

CMB temperature anisotropy via Compton scattering in the epoch of recombination can cause the polarization of photons. One of the usual methods to characterize the polarization state of the radiation field is throughout the Stokes parameters I, Q, U and V. To introduce these parameters in the context of quantum mechanics, one can consider a photon ensemble. The polarization density matrix of photons is defined as

$\rho=\frac{1}{2}\left[\begin{array}{cc}I+Q & U-i V \\ U+i V & I-Q\end{array}\right]$,

where $I$ is the total intensity of radiation, $U, Q$ and $V$ describe the polarization of photons and for unpolarized photons $Q=$ $U=V=0$. The circularly polarized radiation is defined by a non-zero value for $V$ and the linear polarization is described by the Stokes parameters $Q$ and $U$. The parameters $I$ and $V$ are independent of the reference frame whereas $Q$ and $U$ are frame-dependent. Therefore, in the context of cosmology by 
introducing a set of linear combinations of $Q$ and $U$, one can find reference frame independent parameters that are known as $\mathrm{E}$ and $\mathrm{B}$ modes.

Meanwhile, the time evolution of the Stokes parameters can be examined through the Boltzmann equation. This equation provides a systematic way to account for different couplings in a system and is generally expressed as follows

$$
\frac{d f}{d t}=C[f],
$$

where $C[f]$ in the right-hand side of (5) contains all possible collision terms while the left-hand side is known as the Liouville term and involves the effects of gravitational perturbations about the homogeneous cosmology. In the case of photon, the distribution function $f$ is the density matrix $\rho_{i j}$ as is given in (4). Thus the density operator corresponding to the density matrix $\rho_{i j}$ can be given as [31]

$\hat{\rho}=\frac{1}{\operatorname{tr}(\hat{\rho})} \int \frac{d^{3} \mathbf{k}}{(2 \pi)^{3}} \rho_{i j}(\mathbf{k}) a_{i}^{\dagger}(\mathbf{k}) a_{j}(\mathbf{k})$,

and the number operator $D_{i j}^{0}(\mathbf{k})=a_{i}^{\dagger}(\mathbf{k}) a_{j}(\mathbf{k})$, has an expectation value as follows

$$
\left\langle D_{i j}^{0}(\mathbf{k})\right\rangle \equiv \operatorname{tr}\left[\hat{\rho} \hat{D}_{i j}^{0}(\mathbf{k})\right]=(2 \pi)^{3} \delta^{3}(0)\left(2 k^{0}\right) \rho_{i j}(\mathbf{k}) .
$$

However, to examine the time evolution of the photons polarization in the $\mathrm{CMB}$, we need the time evolution of the density matrix. To this end, we substitute (7) in

$\frac{d}{d t} D_{i j}^{0}(\mathbf{k})=i\left[H, D_{i j}^{0}(\mathbf{k})\right]$

where $H$ is the full Hamiltonian, to find the time evolution of $\rho_{i j}$ as

$$
\begin{aligned}
& (2 \pi)^{3} \delta^{3}(0)\left(2 k^{0}\right) \frac{d}{d t} \rho_{i j}(\mathbf{k}) \\
& =i\left\langle\left[H_{I}^{0}(t), D_{i j}^{0}(\mathbf{k})\right]\right\rangle \\
& \quad-\frac{1}{2} \int d t\left\langle\left[H_{I}^{0}(t),\left[H_{I}^{0}(0), D_{i j}^{0}(\mathbf{k})\right]\right]\right\rangle .
\end{aligned}
$$

In (9) $H_{I}^{0}$ is the interacting Hamiltonian at the lowest order [31]. The first and the second term on the right-handed side of (9) are called forward scattering term and higher order collision term, respectively.

\subsection{Dirac fermionic dark matter}

We consider a Dirac fermionic DM which interacts with photon via its magnetic dipole moment with the following Lagrangian $[12,17,47]$
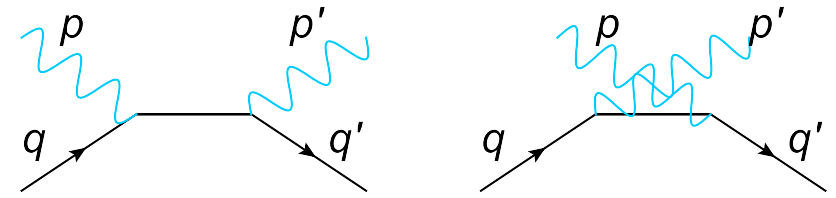

Fig. 1 The typical diagrams for photon-DM scattering

$\mathcal{L}_{\mathrm{DDM}}=-\frac{i}{2} M \bar{\psi} \sigma_{\mu \nu} \psi F^{\mu \nu}$.

The Feynman diagram corresponding to DDM-photon scattering at the lowest order is very similar to the Compton scattering as is shown in Fig. 1. Therefore, the interacting Hamiltonian at the lowest order can be obtained as follows

$$
\begin{aligned}
H_{I}(t)= & -M^{2} \int d^{4} x^{\prime} \int d^{3} \mathbf{x} \bar{\psi}^{-}(x) \sigma^{\mu v} \\
& \times S_{F}\left(x-x^{\prime}\right) \sigma^{\alpha \beta}\left(\partial_{\mu} A_{v}^{-}(x) \partial_{\alpha} A_{\beta}^{+}\left(x^{\prime}\right)\right. \\
& \left.+\partial_{\alpha} A_{\beta}^{-}\left(x^{\prime}\right) \partial_{\mu} A_{v}^{+}(x)\right) \psi^{+}\left(x^{\prime}\right),
\end{aligned}
$$

with the Fourier transformations of the fields and propagator as follows

$$
\begin{aligned}
A_{\mu}(x)= & \int \frac{d^{3} \mathbf{p}}{(2 \pi)^{3} 2 p^{0}} \sum_{s}\left[a_{s}(p) \epsilon_{s \mu}(p) e^{-i p \cdot x}\right. \\
& \left.+a_{s}^{\dagger}(p) \epsilon_{s \mu}^{*}(p) e^{i p . x}\right]
\end{aligned}
$$

$$
\begin{aligned}
\psi(x)= & \int \frac{d^{3} \mathbf{q}}{(2 \pi)^{3}} \frac{m}{q^{0}} \sum_{r}\left[b_{r}(q) u_{r}(q) e^{-i q \cdot x}\right. \\
& \left.+d_{r}^{\dagger}(q) v_{r}(q) e^{i q \cdot x}\right]
\end{aligned}
$$

and

$S_{F}(x)=\int \frac{d^{4} k}{(2 \pi)^{4}} \frac{k+m}{k^{2}-m^{2}+i \theta} e^{-i k \cdot x}$,

where $\epsilon_{s \mu}(p)$ 's are the photon polarization 4-vectors with $s=1,2$ for two physical transverse polarization of a free photon and $a_{s}(p)\left(a_{s}^{\dagger}(p)\right)$ is the annihilation (creation) operator which satisfies the canonical commutation relation as

$\left[a_{s}(p), a_{s^{\prime}}^{\dagger}\left(p^{\prime}\right)\right]=(2 \pi)^{3} 2 p^{0} \delta_{s s^{\prime}} \delta^{(3)}\left(\mathbf{p}-\mathbf{p}^{\prime}\right)$.

In (13) $u_{r}$ and $v_{r}$ are the Dirac spinors, $b_{r}\left(d_{r}\right)$ and $b_{r}^{\dagger}\left(d_{r}^{\dagger}\right)$ are, respectively, the annihilation and creation operators for fermion (antifermion) satisfying

$$
\left\{b_{r}(q), b_{r^{\prime}}^{\dagger}\left(q^{\prime}\right)\right\}=\left\{d_{r}(q), d_{r^{\prime}}^{\dagger}\left(q^{\prime}\right)\right\}=(2 \pi)^{3} \frac{q^{0}}{m} \delta_{r r^{\prime}} \delta^{(3)}\left(\mathbf{q}-\mathbf{q}^{\prime}\right) .
$$


Therefore, the interaction Hamiltonian (11) by using (12), (13) and (14) cast into

$$
\begin{aligned}
H_{I}(t)= & \int d \mathbf{q} d \mathbf{q}^{\prime} d \mathbf{p} d \mathbf{p}^{\prime}(2 \pi)^{3} \delta^{(3)}(\mathbf{q} \\
& \left.+\mathbf{p}-\mathbf{q}^{\prime}-\mathbf{p}^{\prime}\right) e^{i\left(q^{0}+p^{0}-q^{\prime 0}-p^{\prime 0}\right) t} \\
& \times\left[b_{r^{\prime}}^{\dagger}\left(q^{\prime}\right) a_{s^{\prime}}^{\dagger}\left(p^{\prime}\right) \mathcal{M} a_{s}(p) b_{r}(q)\right],
\end{aligned}
$$

where $d \mathbf{q} \equiv \frac{d^{3} \mathbf{q}}{(2 \pi)^{3}} \frac{m}{q^{0}}$ and $d \mathbf{p} \equiv \frac{d^{3} \mathbf{p}}{(2 \pi)^{3}} \frac{1}{2 p^{0}}$ and

$\mathcal{M} \equiv \mathcal{M}_{1}+\mathcal{M}_{2}$,

with

$\mathcal{M}_{1}=M^{2} \frac{\bar{u}_{r^{\prime}}\left(q^{\prime}\right) \notin_{s^{\prime}}\left(p^{\prime}\right) \not p^{\prime}(q+\not p+m) \notin_{s}(p) \not p u_{r}(q)}{2 q \cdot p}$,

and

$\mathcal{M}_{2}=-M^{2} \frac{\bar{u}_{r^{\prime}}\left(q^{\prime}\right) \notin_{s}(p) \not p\left(q-\not p^{\prime}+m\right) \notin_{s^{\prime}}\left(p^{\prime}\right) \not p^{\prime} u_{r}(q)}{2 q \cdot p^{\prime}}$.

Now, we are ready to evaluate the forward scattering term, the first term on the right hand side of (9). To this end, one needs the expectation value of operators such as [31]

$\left\langle a_{1} a_{2} \ldots b_{1} b_{2} \ldots\right\rangle=\left\langle a_{1} a_{2} \ldots\right\rangle\left\langle b_{1} b_{2} \ldots\right\rangle$,

$\left\langle a_{s^{\prime}}^{\dagger}\left(p^{\prime}\right) a_{s}(p)\right\rangle=2 p^{0}(2 \pi)^{3} \delta^{(3)}\left(\mathbf{p}-\mathbf{p}^{\prime}\right) \rho_{s s^{\prime}}(\mathbf{x}, \mathbf{p})$,

and

$\left\langle b_{r^{\prime}}^{\dagger}\left(q^{\prime}\right) b_{r}(q)\right\rangle=(2 \pi)^{3} \delta^{(3)}\left(\mathbf{q}-\mathbf{q}^{\prime}\right) \delta_{r r^{\prime}} n_{\mathrm{DM}}(\mathbf{x}, \mathbf{q})$.

By using the above relations, $\left\langle\left[H_{I}^{0}(0), D_{i j}(\mathbf{k})\right]\right\rangle$ can be obtained as follows

$$
\begin{aligned}
& i\left\langle\left[H_{I}^{0}(0), D_{i j}(\mathbf{k})\right]\right\rangle \\
& \quad=i \int d \mathbf{q} n_{\mathrm{DM}}(\mathbf{x}, \mathbf{q})\left(\delta_{i s} \rho_{s^{\prime} j}(\mathbf{k})-\delta_{j s^{\prime}} \rho_{i s}(\mathbf{k})\right)(2 \pi)^{3} \delta^{(3)}(0) \mathcal{M},
\end{aligned}
$$

where

$$
\mathcal{M}=M^{2} \bar{u}_{r}(q)\left(\notin_{s^{\prime}}(k) \notin_{s}(k)-\phi_{s}(k) \notin_{s^{\prime}}(k)\right) k u_{r}(q),
$$

or

$$
\mathcal{M}=2 M^{2} \epsilon_{s^{\prime}}^{\alpha}(k) \epsilon_{s}^{\beta}(k) k^{\sigma} \epsilon_{\alpha \beta \sigma \lambda} \bar{u}_{r}(q) \gamma^{\lambda} \gamma^{5} u_{r}(q) .
$$

Meanwhile, in the nonrelativistic limit one has [48]

$\bar{u}_{r}(q) \gamma^{0} \gamma^{5} u_{s}(q) \simeq \xi_{r}^{\dagger} \frac{\mathbf{q} \cdot \vec{\sigma}}{m} \xi_{s}$, and

$\bar{u}_{r}(q) \gamma^{i} \gamma^{5} u_{s}(q) \simeq \xi_{r}^{\dagger} \sigma^{i} \xi_{s}$

where $\xi_{r}$ indicates the two component spinor normalized to unity and $\sigma^{i}(i=1,2,3)$ are the Pauli matrices. Therefore, after some manipulations, the amplitude can be rewritten as

$\mathcal{M} \simeq 2 M^{2}\left(\mathbf{k} \cdot\left(\vec{\epsilon}_{s^{\prime}} \times \vec{\epsilon}_{s}\right) \xi_{r}^{\dagger} \frac{\mathbf{q} \cdot \vec{\sigma}}{m} \xi_{r}-k^{0}\left(\vec{\epsilon}_{s^{\prime}} \times \vec{\epsilon}_{s}\right) \cdot \xi_{r}^{\dagger} \vec{\sigma} \xi_{r}\right)$.

Using the following relations

$\xi_{r}^{\dagger} \mathbf{q} \cdot \vec{\sigma} \xi_{r}=(-1)^{r+1}|\mathbf{q}|$,

$\xi_{r}^{\dagger} \vec{\sigma} \xi_{r}=(-1)^{r+1} \hat{\mathbf{q}}$,

we find that, unfortunately, (29) after averaging on both helicities of the Dirac fermions, leads to a vanishing average as

$\frac{1}{2} \sum_{r}\left(2 M^{2}\left\{\mathbf{k} \cdot\left(\vec{\epsilon}_{s^{\prime}} \times \vec{\epsilon}_{s}\right) \xi_{r}^{\dagger} \frac{\mathbf{q} \cdot \vec{\sigma}}{m} \xi_{r}-k^{0}\left(\vec{\epsilon}_{s^{\prime}} \times \vec{\epsilon}_{s}\right) \cdot \xi_{r}^{\dagger} \vec{\sigma} \xi_{r}\right\}\right)=0$,

where $r$ stands for the helicity. Therefore, the photon-DM forward scattering for Dirac fermions with both chiralities has not any contribution on the CMB polarization.

\subsection{Majorana dark matter: transition magnetic dipole moment}

In this section, we are going to investigate the effect of DDMphoton interaction on the CMB polarization through Majorana magnetic moment.

Two singlet Majorana fermions $\chi_{1}$ and $\chi_{2}$, with mass splitting $\delta$, can interact with photons via transition magnetic moment based on the following Lagrangian [49]

$\mathcal{L}_{\mathrm{DDM}}=-\frac{i}{2} M_{12} \bar{\chi}_{1} \sigma_{\mu \nu} \chi_{2} F^{\mu \nu}+$ H.C.

In (33) the $\chi_{i}$ 's can be considered as $\left(\chi=\psi_{R}+\psi_{R}^{c}\right)$ or $\left(\chi=\psi_{L}+\psi_{L}^{c}\right)$ or even both together. However, in the latter case the contribution of the forward scattering on the CMB polarization, as the largest term, is zero when the coupling $M_{12}$ for both chiralities is considered to be equal, see appendix A. In fact, existing asymmetry in the distribution function of particles which interact with an ensemble of photons can make a net polarized state. Since in the case of Majorana particles with the same coupling for both chiralities as well as Dirac fermions there is no such asymmetry in the distribution function, these types 
of interaction can not affect the CMB polarization. Meanwhile, in the case of Majorana particles, which can couple to photon through just one kind of chiralities one can expect to get a net polarization. Nevertheless, we will consider the Majorana fermions to be the right handed neutrinos $\left(\chi=\psi_{R}+\psi_{R}^{c}\right)$ which cast the Lagrangian (33) into

$$
\begin{aligned}
\mathcal{L}_{\mathrm{DDM}}= & -\frac{i}{2} M_{12}\left(\bar{\psi}^{c}{ }_{1} \sigma_{\mu \nu} P_{R} \psi_{2} F^{\mu \nu}\right. \\
& \left.+\bar{\psi}_{1} \sigma_{\mu \nu} P_{L} \psi_{2}^{c} F^{\mu \nu}\right)+ \text { H.C. },
\end{aligned}
$$

where $P_{R}=\frac{1}{2}\left(1+\gamma^{5}\right), P_{L}=\frac{1}{2}\left(1-\gamma^{5}\right)$ and $\psi^{c}=$ $-i \gamma_{2} \psi^{\star}$.

In the following, first, we will assume that the two singlet fermions are both stable DM particles. Next, we will suppose that the heavier Majorana particle is not stable and decays into the lighter one which is stable and acts as a Majorana DM.

\section{-Stable majorana particle :}

First, we consider two singlet Majorana particles with masses $m_{\mathrm{DM}_{1}}$ and $m_{\mathrm{DM}_{2}}$ act as DM. Based on (34) and by using (12), (13), (14) and the fact that $S_{\mathrm{F}}^{c}(k)=S_{F}(-k)$ [50], the total Feynman amplitude corresponding to DDMphoton scattering at the lowest order is obtained from the sum of all Feynman diagrams in Fig. 1 as follows

$\mathcal{M}^{\prime}=\mathcal{M}_{1}^{\prime}+\mathcal{M}_{2}^{\prime}$

where

$$
\begin{aligned}
\mathcal{M}_{1}^{\prime}= & \frac{-1}{2} M_{12}^{2}\left(\frac{\bar{u}_{r^{\prime}}\left(q^{\prime}\right) \notin_{s^{\prime}}\left(p^{\prime}\right) \not p^{\prime} P_{L}\left(q+\not p-m_{\mathrm{DM}_{2}}\right) \notin_{s}(p) \not p P_{R} u_{r}(q)}{(p+q)^{2}-m_{\mathrm{DM}_{2}}^{2}}\right. \\
& +\frac{\bar{u}_{r^{\prime}}\left(q^{\prime}\right) \notin_{s}(p) \not p P_{L}\left(q-\not p^{\prime}-m_{\mathrm{DM}_{2}}\right) \notin_{s^{\prime}}\left(p^{\prime}\right) \not p^{\prime} P_{R} u_{r}(q)}{\left(-q+p^{\prime}\right)^{2}-m_{\mathrm{DM}_{2}}^{2}} \\
& +\frac{\bar{v}_{r}(q) \notin_{s}(p) \not p P_{R}\left(-q+\not p+m_{\mathrm{DM}_{2}}\right) \notin_{s^{\prime}}\left(p^{\prime}\right) \not p^{\prime} P_{L} v_{r^{\prime}}\left(q^{\prime}\right)}{(p-q)^{2}-m_{\mathrm{DM}_{2}}^{2}} \\
& \left.+\frac{\bar{v}_{r}(q) \notin_{s^{\prime}}\left(p^{\prime}\right) \not p^{\prime} P_{R}\left(-q^{\prime}-\not p+m_{\mathrm{DM}_{2}}\right) \notin_{s}(p) \not p P_{L} v_{r^{\prime}}\left(q^{\prime}\right)}{\left(-q^{\prime}-p\right)^{2}-m_{\mathrm{DM}_{2}}^{2}}\right),
\end{aligned}
$$

and $\mathcal{M}_{2}^{\prime}$ is the same as $\mathcal{M}_{1}^{\prime}$ except that the indices 1 and 2 are replaced. To evaluate the forward scattering term, one needs to calculate $\left\langle\left[H_{I}^{0}(0), D_{i j}(\mathbf{k})\right]\right\rangle$ as follows

$$
\begin{aligned}
i\left\langle\left[H_{I}^{0}(0), D_{i j}(\mathbf{k})\right]\right\rangle= & i \int d \mathbf{q} n_{D M}(\mathbf{x}, \mathbf{q})\left(\delta_{i s} \rho_{s^{\prime} j}(\mathbf{k})\right. \\
& \left.-\delta_{j s^{\prime}} \rho_{i s}(\mathbf{k})\right)(2 \pi)^{3} \delta^{(3)}(0) \mathcal{M}^{\prime},
\end{aligned}
$$

where

$$
\mathcal{M}^{\prime}=\left.\mathcal{M}^{\prime}\right|_{q^{\prime}=q, p^{\prime}=p=k, r=r^{\prime}}
$$

By imposing the conditions $q^{\prime}=q$ and $p^{\prime}=p=k$, (35) cast into the following relation

$$
\begin{aligned}
\mathcal{M}^{\prime}= & \frac{-1}{2} \frac{M_{12}^{2}}{(2 k \cdot q)^{2}-\left(m_{\mathrm{DM}_{2}}^{2}-m_{\mathrm{DM}_{1}}^{2}\right)^{2}} \\
& \times\left(\left(2 k \cdot q+\left(m_{\mathrm{DM}_{2}}^{2}-m_{\mathrm{DM}_{1}}^{2}\right)\right)(2 k \cdot q)\right. \\
& \times \bar{u}_{r}(q) \notin_{s^{\prime}}(k) \notin_{s}(k) k P_{R} u_{r}(q) \\
& -\left(2 k \cdot q-\left(m_{\mathrm{DM}_{2}}^{2}-m_{\mathrm{DM}_{1}}^{2}\right)\right)(2 k \cdot q) \\
& \times \bar{u}_{r}(q) \notin_{s}(k) \notin_{s^{\prime}}(k) k P_{R} u_{r}(q) \\
& +\left(2 k \cdot q+\left(m_{\mathrm{DM}_{2}}^{2}-m_{\mathrm{DM}_{1}}^{2}\right)\right)(2 k \cdot q) \\
& \times \bar{v}_{r}(q) \notin_{s}(k) \notin_{s^{\prime}}(k) k P_{L} v_{r}(q) \\
& -\left(2 k \cdot q-\left(m_{\mathrm{DM}_{2}}^{2}-m_{\mathrm{DM}_{1}}^{2}\right)\right)(2 k \cdot q) \bar{v}_{r}(q) \notin_{s^{\prime}}(k) \notin_{s}(k) k k \\
& \left.\times P_{L} v_{r}(q)\right)+(1 \leftrightarrow 2) .
\end{aligned}
$$

In the above relation, terms proportional to the mass difference lead to the product of polarization vectors, $\epsilon_{s} \cdot \epsilon_{s^{\prime}}=$ $-\delta_{s s^{\prime}}$, and therefore their contributions will be zero. For the remaining terms, we use the identity

$\bar{u}_{r}(q) \gamma^{\lambda} u_{s}(q)=\bar{v}_{r}(q) \gamma^{\lambda} v_{s}(q)=\frac{q^{\lambda}}{m_{\mathrm{DM}}} \delta_{r s}$

and arrive at the following relation

$$
\begin{aligned}
\mathcal{M}^{\prime}= & -M_{12}^{2} \frac{(2 k \cdot q)^{2}}{(2 k \cdot q)^{2}-\left(m_{\mathrm{DM}_{2}}^{2}-m_{\mathrm{DM}_{1}}^{2}\right)^{2}} \epsilon_{\alpha \beta \sigma \lambda} \epsilon_{s^{\prime}}^{\alpha}(k) \\
& \times \epsilon_{s}^{\beta}(k) k^{\sigma} \frac{q^{\lambda}}{m_{\mathrm{DM}_{1}}}+(1 \leftrightarrow 2),
\end{aligned}
$$

and hence, in the non-relativistic limit, the total Feynman amplitude is given by

$$
\begin{aligned}
\mathcal{M}^{\prime}= & -M_{12}^{2} \frac{(2 k \cdot q)^{2}}{(2 k \cdot q)^{2}-\left(m_{\mathrm{DM}_{2}}^{2}-m_{\mathrm{DM}_{1}}^{2}\right)^{2}}\left(\mathbf{k} \cdot\left(\vec{\epsilon}_{s^{\prime}} \times \vec{\epsilon}_{s}\right)\right. \\
& \left.-k^{0} v\left(\vec{\epsilon}_{s^{\prime}} \times \vec{\epsilon}_{s}\right) \cdot \hat{\mathbf{v}}\right)+(1 \leftrightarrow 2),
\end{aligned}
$$

where $v=|\vec{q}| / m_{\mathrm{DM}}$.

\subsubsection{Case $\delta=m_{D M_{2}}-m_{D M_{1}} \ll k^{0}$}

Here, we consider the case that $\delta=m_{\mathrm{DM}_{2}}-m_{\mathrm{DM}_{1}} \ll k^{0}$ and therefore (42) can be estimated as follows

$\mathcal{M}^{\prime} \simeq-M_{12}^{2} \mathbf{k} \cdot\left(\vec{\epsilon}_{s^{\prime}} \times \vec{\epsilon}_{s}\right)+(1 \leftrightarrow 2)$.

Although the second term in (42) is similar to the first one, a straightforward calculation leads to a negligible value for this term. In fact, the order of the second term is smaller than the first one due to the presence of $v$ and therefore we ignore the terms propotional to the DM velocity of (42). Now by 
substituting (43) in (37) and using (9), the time evolution of density matrix element can be written as

$$
\begin{aligned}
\frac{d \rho_{i j}}{d t}= & -i M^{2}\left(n_{\mathrm{DM}_{1}}(\mathbf{x})+n_{\mathrm{DM}_{2}}(\mathbf{x})\right)\left(\delta_{i s} \rho_{s^{\prime} j}(\mathbf{k})\right. \\
& \left.-\delta_{j s^{\prime}} \rho_{i s}(\mathbf{k})\right)\left(\vec{\epsilon}_{s^{\prime}} \times \vec{\epsilon}_{s}\right) \cdot \hat{\mathbf{k}},
\end{aligned}
$$

where $M_{12}^{2}=M_{21}^{2}=M^{2}, \hat{\mathbf{k}}=\mathbf{k} / k^{0}$ and the DM number density $n_{\mathrm{DM}_{\mathrm{i}}}(\mathrm{i}=1,2)$ is

$n_{\mathrm{DM}_{\mathrm{i}}}(\mathbf{x})=\int \frac{d^{3} \mathbf{q}}{(2 \pi)^{3}} n_{\mathrm{DM}_{\mathrm{i}}}(\mathbf{x}, \mathbf{q})$.

Since $n_{\mathrm{DM}_{1}}(\mathbf{x})+n_{\mathrm{DM}_{2}}(\mathbf{x})=n_{\mathrm{DM}}(\mathbf{x}),(44)$ cast into

$\frac{d \rho_{i j}}{d t}=-i M^{2} n_{\mathrm{DM}}(\mathbf{x})\left(\delta_{i s} \rho_{s^{\prime} j}(\mathbf{k})-\delta_{j s^{\prime}} \rho_{i s}(\mathbf{k})\right)\left(\vec{\epsilon}_{s^{\prime}} \times \vec{\epsilon}_{s}\right) \cdot \hat{\mathbf{k}}$,

and consequently, the Stokes parameters evolve as

$\frac{d I}{d t}=C_{e \gamma}^{I}$,

$\frac{d}{d t}(Q \pm i U)=C_{e \gamma}^{ \pm} \mp i \dot{\tau}_{\mathrm{DM}}(Q \pm i U)$,

$\frac{d V}{d t}=C_{e \gamma}^{V}$,

where $C_{e \gamma}^{I}, C_{e \gamma}^{V}$ and $C_{e \gamma}^{ \pm}$show the contribution of Thomson scattering [31] and $\dot{\tau}_{\mathrm{DM}}$ is defined as follows

$\dot{\tau}_{\mathrm{DM}}=\frac{3}{8 \pi}\left(\frac{m_{e}}{\alpha}\right)^{2} \sigma_{T} M^{2} n_{\mathrm{DM}}$,

where $\sigma_{T}$ is the Thomson cross section. The second term in the right handed side of (48) shows that the photon-DDM forward scattering affects the time evolution of the Stokes parameters $Q$ and $U$. In the approximation that $\delta$ is small, $\left(m_{\mathrm{DM}_{1}} \approx m_{\mathrm{DM}_{2}} \approx m_{\mathrm{DM}}\right)$, and by using the relation between magnetic dipole moment $M$ and DM-photon scattering cross section $\langle\sigma v\rangle[12,13]$

$\langle\sigma v\rangle \approx \frac{1}{2 \pi} M^{4} m_{\mathrm{DM}}^{2}$,

(50) cast into

$\dot{\tau}_{\mathrm{DM}}=\frac{3}{8 \pi}\left(\frac{m_{\mathrm{e}}}{m_{\mathrm{DM}}}\right)^{2} \frac{\sigma_{T}}{\alpha^{2}} \sqrt{2 \pi\langle\sigma v\rangle} \rho_{\mathrm{DM}}$,

where $\rho_{\mathrm{DM}}$ is the DM mass density. To compare the contribution of the CMB-DDM interaction with respect to the Compton scattering, we need to have the ratio $\frac{\dot{t}_{\mathrm{DM}}}{\dot{\tau}_{\mathrm{e}}}$ where $\dot{\tau}_{\mathrm{e}}$ is the differential optical depth for Compton scattering which is denoted by

$\dot{\tau}_{\mathrm{e}}=a(\eta) \sigma_{T} n_{\mathrm{e}}$, where $\eta$ is the conformal time and $a(\eta)$ is the expansion factor which is normalized to unity for present time $\left(\eta=\eta_{0}\right)$. Hence, the ratio of $\dot{\tau}_{\text {DM }}$ with respect to the $\dot{\tau}_{\mathrm{e}}$, at the present time, can be found as

$$
\frac{\dot{\tau}_{\mathrm{DM}}}{\dot{\tau}_{\mathrm{e}}}=\frac{3}{8 \pi}\left(\frac{m_{\mathrm{e}}}{m_{\mathrm{DM}}}\right)^{2} \frac{\Omega_{\mathrm{DM}}}{\Omega_{\mathrm{BM}}} \frac{m_{\mathrm{p}}}{\alpha^{2}} \sqrt{2 \pi\langle\sigma v\rangle},
$$

where $\Omega_{\mathrm{DM}}$ and $\Omega_{\mathrm{BM}}$ are the DM density parameter and the baryonic matter density parameter, respectively. To arrive the above relation we have used the fact that the number density of electron is equal to the number density of proton and it is approximately equal to the baryonic matter number density

$n_{\mathrm{e}}=n_{\mathrm{p}} \approx n_{\mathrm{BM}}$.

However, the value of the ratio given in (54) can be estimated as

$$
\begin{aligned}
\frac{\dot{\tau}_{\mathrm{DM}}}{\dot{\tau}_{e}} \simeq & 5.2 \times 10^{-11}\left(\frac{m_{\mathrm{DM}}}{10 \mathrm{GeV}}\right)^{-2}\left(\frac{\langle\sigma v\rangle}{\left(10^{-30}\right) \frac{\mathrm{cm}^{3}}{\mathrm{~s}}}\right)^{1 / 2} \\
& \times\left(\frac{\Omega_{\mathrm{DM}}}{0.26}\right)\left(\frac{\Omega_{\mathrm{BM}}}{0.04}\right)^{-1}\left(\frac{m_{\mathrm{p}}}{1 \mathrm{GeV}}\right),
\end{aligned}
$$

where for DM particles with masses $10 \mathrm{GeV}$--- $10 \mathrm{MeV}$ varies as $5.2 \times 10^{-11}---5.2 \times 10^{-5}$.

\subsubsection{Case $\delta=m_{D M_{2}}-m_{D M_{1}} \gg k^{0}$}

In this case, (42) can be estimated as follows

$\mathcal{M}^{\prime} \simeq M_{12}^{2} \frac{(2 k \cdot q)^{2}}{\left(m_{\mathrm{DM}_{2}}^{2}-m_{\mathrm{DM}_{1}}^{2}\right)^{2}} \mathbf{k} \cdot\left(\vec{\epsilon}_{s^{\prime}} \times \vec{\epsilon}_{s}\right)+(1 \leftrightarrow 2)$.

In the nonrelativistic limit and if we assume $m_{\mathrm{DM}_{1}}$ is the same order of $m_{\mathrm{DM}_{2}}$ we will have

$\mathcal{M}^{\prime} \simeq M_{12}^{2}\left(\frac{k^{0}}{\delta}\right)^{2} \mathbf{k} \cdot\left(\vec{\epsilon}_{s^{\prime}} \times \vec{\epsilon}_{s}\right)+(1 \leftrightarrow 2)$.

For the cases in which $k^{0} \ll \delta \ll m_{\mathrm{DM}},\left(m_{\mathrm{DM}_{1}} \approx m_{\mathrm{DM}_{2}} \approx\right.$ $m_{\mathrm{DM}}$ ), and after some calculation, one can find the evolution of the Stokes parameters similar to (47-49) except that $\dot{\tau}_{\mathrm{DM}}$ is defined as follows

$\dot{\tau}_{\mathrm{DM}}=\left(\frac{k^{0}}{\delta}\right)^{2} \frac{3}{8 \pi}\left(\frac{m_{\mathrm{e}}}{m_{\mathrm{DM}}}\right)^{2} \frac{\sigma_{T}}{\alpha^{2}} \sqrt{2 \pi\langle\sigma v\rangle} \rho_{\mathrm{DM}}$,

which leads to

$$
\frac{\dot{\tau}_{\mathrm{DM}}}{\dot{\tau}_{\mathrm{e}}}=\frac{3}{8 \pi}\left(\frac{k^{0}}{\delta}\right)^{2}\left(\frac{m_{\mathrm{e}}}{m_{\mathrm{DM}}}\right)^{2} \frac{\Omega_{\mathrm{DM}}}{\Omega_{\mathrm{BM}}} \frac{m_{\mathrm{p}}}{\alpha^{2}} \sqrt{2 \pi\langle\sigma v\rangle} .
$$


The above relation clearly shows that the contribution of the photon-DDM scattering on the CMB polarization in the case in which $\delta \gg k^{0}$ will be negligible and therefore we will not consider this case for the rest of the paper.

-Unstable majorana particles :

Now we investigate the possibility that the heavier Majorana particle (with mass $m_{\mathrm{DM}_{2}}$ ) can decay into the lighter one (with mass $m_{\mathrm{DM}_{1}}$ ) which is stable. In this case, the Feynman amplitude corresponding to DDM-photon scattering will be the same as (36) which after imposing the conditions $q^{\prime}=q$ and $p^{\prime}=p=k$ due to (37) and (38) cast into

$$
\begin{aligned}
\mathcal{M}^{\prime}= & -M_{12}^{2} \frac{(2 k \cdot q)^{2}}{(2 k \cdot q)^{2}-\left(m_{\mathrm{DM}_{2}}^{2}-m_{\mathrm{DM}_{1}}^{2}\right)^{2}} \\
& \times \epsilon_{\alpha \beta \sigma \lambda} \epsilon_{s^{\prime}}^{\alpha}(k) \epsilon_{s}^{\beta}(k) k^{\sigma} \frac{q^{\lambda}}{m_{\mathrm{DM}_{1}}} .
\end{aligned}
$$

The same as the previous part, we work in two approximation: First, we consider $\delta=m_{\mathrm{DM}_{2}}-m_{\mathrm{DM}_{1}} \ll k^{0}$. In the nonrelativistic limit, the time evolution of density matrix element will be obtained as (46)

$$
\frac{d \rho_{i j}}{d t}=-i M^{2} n_{\mathrm{DM}}(\mathbf{x})\left(\delta_{i s} \rho_{s^{\prime} j}(\mathbf{k})-\delta_{j s^{\prime}} \rho_{i s}(\mathbf{k})\right)\left(\vec{\epsilon}_{s^{\prime}} \times \vec{\epsilon}_{s}\right) \cdot \hat{\mathbf{k}}
$$

where $M_{12}=M$ and $n_{\mathrm{DM}_{1}}=n_{\mathrm{DM}}$. Therefore the results will be the same as the previous part and the contribution of this interaction on the polarization of the CMB will be determined through (52). However, in the case of decaying heavier Majorana particle into the lighter one, we have more constraints on $\delta$ due to the life time of the heavier particle which depends on the mass splitting as follows

$\tau\left(\chi_{2} \rightarrow \chi_{1} \gamma\right)=\frac{\pi}{M^{2}} \frac{1}{\delta^{3}}$.

Based on this relation, for a small value of the mass splitting, the value of the magnetic moment must be large enough to occur the decay process within the cosmological timescale and for a small value of the magnetic moment, the decay can not occur. Meanwhile, for a large value of the mass splitting $\left(\delta \gg k^{0}\right)$ the heavier particle can decay but the contribution on the CMB polarization will be suppressed as $\left(\frac{k^{0}}{\delta}\right)^{2}$.

\section{Generalized Boltzmann equation for the CMB}

The CMB polarization pattern includes two types of polarization, E and B-modes. While the E-mode polarization can be produced in the presence of scalar perturbations, the B-mode polarization is only generated by tensor perturbations. However, in the previous section, we showed that photon-DDM interaction can act as a source for generating the B mode polarization pattern in the presence of scalar perturbations. In fact, in the case that the mass spilitting of Majorana DM is smaller than the energy of the CMB photon, this interaction can modify the power spectrum of the B-mode polarization.

The CMB radiation transfer is described by the multipole moments of temperature (I) and polarization (P) $[34,35]$

$\Delta_{\mathrm{I}, \mathrm{P}}^{\mathrm{S}}(\eta, K, \mu)=\sum_{l=0}^{\infty}(2 l+1)(-i)^{l} \Delta_{\mathrm{I}, \mathrm{P}_{l}}^{\mathrm{S}}(\eta, K) P_{l}(\mu)$,

where $P_{l}(\mu)$ is the Legendre polynomial of rank $l, \mu=$ $\hat{\mathbf{n}} . \hat{\mathbf{K}}=\cos \theta$ and $\theta$ is the angle between the CMB photon direction $\hat{\mathbf{n}}=\frac{\mathbf{k}}{|\mathbf{k}|}$ and the wave vectors $\mathbf{K}$ of the Fourier modes of scalar perturbations (S). Since for a given Fourier mode, one can choose a coordinate system in which $\mathbf{K} \| \hat{\mathbf{z}}$ then the Boltzmann equation in the presence of Thomson scattering and DDM-photon interaction can be written as

$$
\begin{aligned}
& \frac{d}{d \eta} \Delta_{\mathrm{I}}^{\mathrm{S}}+i K \mu \Delta_{\mathrm{I}}^{\mathrm{S}}+4[\dot{\Psi}-i K \mu \Phi] \\
& \quad=\dot{\tau}_{\mathrm{e}}\left[-\Delta_{\mathrm{I}}^{\mathrm{S}}+\Delta_{\mathrm{I}}^{\mathrm{S} \circ}+i \mu v_{b}+\frac{1}{2} P_{2}(\mu) \Pi\right] \\
& \frac{d}{d \eta} \Delta_{\mathrm{P}}^{ \pm \mathrm{S}}+i K \mu \Delta_{\mathrm{P}}^{ \pm \mathrm{S}}=\dot{\tau}_{\mathrm{e}}\left[-\Delta_{\mathrm{P}}^{ \pm \mathrm{S}}-\frac{1}{2}\left[1-P_{2}(\mu)\right] \Pi\right] \\
& \quad \mp i a(\eta) \dot{\tau}_{\mathrm{DM}} \Delta_{\mathrm{P}}^{ \pm \mathrm{S}}
\end{aligned}
$$

where $\Psi$ and $\Phi$ are the metric perturbations, $\eta$ is the conformal time, $a(\eta)$ is the expansion factor which is normalized to unity for present time $\left(\eta=\eta_{0}\right)$ and $v_{b}$ is the baryon bulk velocity, $\Pi \equiv \Delta_{\mathrm{I}}^{\mathrm{S}_{2}}+\Delta_{\mathrm{P}}^{\mathrm{S}_{2}}-\Delta_{\mathrm{P}}^{\mathrm{S}_{\circ}}$ and the polarization anisotropy is given by

$\Delta_{\mathrm{P}}^{ \pm \mathrm{S}}=Q^{\mathrm{S}} \pm i U^{\mathrm{S}}$

which can cast the equation of polarization anisotropy into $[37,38]$

$\frac{d}{d \eta}\left[\Delta_{\mathrm{P}}^{ \pm \mathrm{S}} e^{i K \mu \eta \pm i \tilde{\tau}_{\mathrm{DM}}+\tilde{\tau}_{\mathrm{e}}}\right]=-\frac{1}{2} e^{i K \mu \eta \pm i \tilde{\tau}_{\mathrm{DM}}+\tilde{\tau}_{\mathrm{e}}} \dot{\tau}_{\mathrm{e}}\left[1-P_{2}(\mu)\right] \Pi$,

where

$\tilde{\tau}_{\mathrm{DM}}(\eta, \mu) \equiv \int_{0}^{\eta} d \eta a(\eta) \dot{\tau}_{\mathrm{DM}}, \quad \tilde{\tau}_{\mathrm{e}}(\eta) \equiv \int_{0}^{\eta} d \eta a(\eta) \dot{\tau}_{\mathrm{e}}$.

Now by integrating (68) along the line of sight up to the present time $\eta_{0}$, with the initial condition $\Delta_{\mathrm{P}}^{ \pm \mathrm{S}}(0, K, \mu)=0$, yields 
$\Delta_{\mathrm{P}}^{ \pm \mathrm{S}}\left(\eta_{0}, K, \mu\right)=\frac{3}{4}\left(1-\mu^{2}\right) \int_{0}^{\eta_{\circ}} d \eta e^{i x \mu \pm i \tau_{\mathrm{DM}}(\eta)-\tau_{\mathrm{e}}} \dot{\tau}_{\mathrm{e}} \Pi(\eta, K)$,

where $x=K\left(\eta_{0}-\eta\right)$ and

$\tau_{\mathrm{DM}}(\eta)=\int_{\eta}^{\eta_{0}} d \eta a(\eta) \dot{\tau}_{\mathrm{DM}}(\eta)=\int_{\eta}^{\eta_{0}} d \eta a(\eta) \sqrt{2 \pi\langle\sigma v\rangle} \frac{\rho_{\mathrm{DM}}}{m_{\mathrm{DM}}^{2}}$,

or in terms of the redshift $z$

$\tau_{\mathrm{DM}}(z)=\frac{\sqrt{2 \pi\langle\sigma v\rangle}}{m_{\mathrm{DM}}^{2}} \int_{0}^{z} d z^{\prime} \rho_{\mathrm{DM}}^{0} \frac{\left(1+z^{\prime}\right)^{2}}{H\left(z^{\prime}\right)}$.

To obtain (72) from (71), we have used

$\rho_{\mathrm{DM}}=\rho_{\mathrm{DM}}^{0}(1+z)^{3}$,

where $\rho_{\mathrm{DM}}^{0}$ is mass density of DM in present time and $a d \eta=$ $-\frac{d z}{H(z)(1+z)}$ where $H(z)$ can be obtained from Friedmann equation in the matter dominated era as follows

$\frac{H^{2}}{H_{0}^{2}}=\Omega_{M}^{0}(1+z)^{3}+\Omega_{\Lambda}^{0}$,

where $H_{0} \approx 67 \mathrm{kms}^{-1} \mathrm{Mpc}^{-1}, \Omega_{M}^{0} \approx 0.31, \Omega_{\Lambda}^{0} \approx 0.69$ [51].

Meanwhile E-mode and B-mode polarizations can be defined in terms of $\Delta_{\mathrm{P}}^{ \pm \mathrm{S}}\left(\eta_{0}, K, \mu\right)$ as follows [31,33-35]

$\Delta_{\mathrm{E}}^{(\mathrm{S})}\left(\eta_{0}, K, \mu\right) \equiv-\frac{1}{2}\left[\bar{\partial}^{2} \Delta_{\mathrm{P}}^{+(\mathrm{S})}\left(\eta_{0}, K, \mu\right)+\partial^{2} \Delta_{\mathrm{P}}^{-(\mathrm{S})}\left(\eta_{0}, K, \mu\right)\right]$,

$\Delta_{\mathrm{B}}^{\mathrm{S}}\left(\eta_{0}, K, \mu\right) \equiv \frac{i}{2}\left[\bar{\partial}^{2} \Delta_{\mathrm{P}}^{+\mathrm{S}}\left(\eta_{0}, K, \mu\right)-\partial^{2} \Delta_{\mathrm{P}}^{-\mathrm{S}}\left(\eta_{\circ}, K, \mu\right)\right]$,

where $\partial$ and $\bar{\partial}$ are spin raising and lowering operators, respectively. Thus by assuming the scalar perturbation to be axially symmetric around $\mathbf{K}$ one has

$$
\begin{aligned}
& \bar{\partial}^{2} \Delta_{\mathrm{P}}^{+\mathrm{S}}\left(\eta_{0}, K, \mu\right)=\partial_{\mu}^{2}\left[\left(1-\mu^{2}\right) \Delta_{\mathrm{P}}^{+\mathrm{S}}\left(\eta_{0}, K, \mu\right)\right], \\
& \partial^{2} \Delta_{\mathrm{P}}^{-\mathrm{S}}\left(\eta_{0}, K, \mu\right)=\partial_{\mu}^{2}\left[\left(1-\mu^{2}\right) \Delta_{\mathrm{P}}^{-\mathrm{S}}\left(\eta_{0}, K, \mu\right)\right],
\end{aligned}
$$

which can cast (75) and (76) into

$\Delta_{\mathrm{E}}^{\mathrm{S}}\left(\eta_{0}, K, \mu\right)=-\frac{3}{4} \int_{0}^{\eta_{0}} d \eta g_{\mathrm{e}}(\eta) \Pi(\eta, K) \partial_{\mu}^{2}\left[\left(1-\mu^{2}\right) e^{i x \mu} \cos \tau_{\mathrm{DM}}\right]$,

$\Delta_{\mathrm{B}}^{\mathrm{S}}\left(\eta_{0}, K, \mu\right)=\frac{3}{4} \int_{0}^{\eta_{0}} d \eta g_{\mathrm{e}}(\eta) \Pi(\eta, K) \partial_{\mu}^{2}\left[\left(1-\mu^{2}\right) e^{i x \mu} \sin \tau_{\mathrm{DM}}\right]$,

where $g_{\mathrm{e}}(\eta)=\dot{\tau}_{\mathrm{e}} e^{-\tau_{\mathrm{e}}}$ is the visibility function of electron. As one can easily see for $\tau_{\mathrm{DM}} \neq 0$ the equations (79) and
(80) show that the DDM-photon interaction produces the nontrivial B-mode polarization and modify of the ordinary E-mode polarization. However, the power spectrum for the $\mathrm{E}$ and B-modes can be obtained by integrating over the initial power spectrum of the metric perturbation as [31,33-35]

$$
\begin{aligned}
C_{l}^{\mathrm{EE}, \mathrm{S}}= & \frac{1}{2 l+1} \frac{(l-2) !}{(l+2) !} \\
& \times \int d^{3} K P_{\mathrm{S}}(K)\left|\sum_{m} \int d \Omega Y_{l m}^{*}(\mathbf{n}) \Delta_{\mathrm{E}}^{\mathrm{S}}\left(\eta_{0}, K, \mu\right)\right|^{2}, \\
C_{l}^{\mathrm{BB}, \mathrm{S}}= & \frac{1}{2 l+1} \frac{(l-2) !}{(l+2) !} \\
& \times \int d^{3} K P_{\mathrm{S}}(K)\left|\sum_{m} \int d \Omega Y_{l m}^{*}(\mathbf{n}) \Delta_{\mathrm{B}}^{\mathrm{S}}\left(\eta_{0}, K, \mu\right)\right|^{2},
\end{aligned}
$$

where $P_{\mathrm{S}}(K)$ is the initial power spectrum of the scalar mode perturbation. By using identities

$\partial_{\mu}^{2}\left(1-\mu^{2}\right) e^{i x \mu} \equiv\left(1+\partial_{x}^{2}\right) x^{2} e^{i x \mu}$,

and

$\int d \Omega Y_{l m}^{*} e^{i x \mu}=(i)^{l} \sqrt{4 \pi(2 l+1) j_{l}(x)} \delta_{m 0}$,

the equations (81) and (82) can be rewritten as

$$
\begin{aligned}
C_{l}^{\mathrm{EE}, \mathrm{S}}= & (4 \pi)^{2} \frac{(l+2) !}{(l-2) !} \\
& \times \int d^{3} K P_{\mathrm{S}}(K)\left|\frac{3}{4} \int_{0}^{\eta_{0}} d \eta g_{\mathrm{e}}(\eta) \Pi(\eta, K) \frac{j_{l}}{x^{2}} \cos \tau_{\mathrm{DM}}\right|^{2}, \\
C_{l}^{\mathrm{BB}, \mathrm{S}}= & (4 \pi)^{2} \frac{(l+2) !}{(l-2) !} \\
& \times \int d^{3} K P_{\mathrm{S}}(K)\left|\frac{3}{4} \int_{0}^{\eta_{0}} d \eta g_{\mathrm{e}}(\eta) \Pi(\eta, K) \frac{j_{l}}{x^{2}} \sin \tau_{\mathrm{DM}}\right|^{2} .
\end{aligned}
$$

We have numerically calculated the B-mode power spectra using CMBquick code for different values of $\langle\sigma v\rangle, m_{\mathrm{DM}}$ and $r$-parameter as are shown in Figs. 2 and 3. However, to see how the curves depend on DM-photon interaction one can approximate (85) and (86) as follows

$C_{l}^{\mathrm{EE}, \mathrm{S}}=\bar{C}_{l}^{\mathrm{EE}, \mathrm{S}}\left(\cos ^{2} \bar{\tau}_{\mathrm{DM}}\right)$,

and

$C_{l}^{\mathrm{BB}, \mathrm{S}}=\bar{C}_{l}^{\mathrm{EE}, \mathrm{S}}\left(\sin ^{2} \bar{\tau}_{\mathrm{DM}}\right)$, 


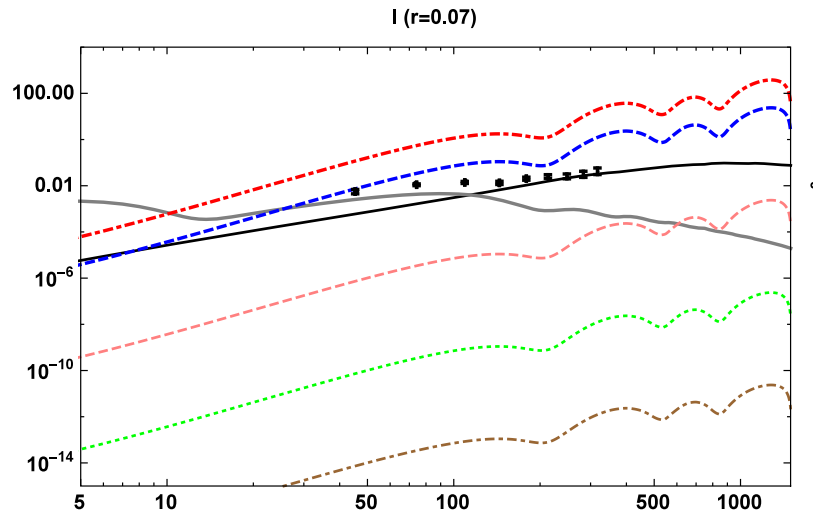

(a) To plot above curves, $\langle\sigma v\rangle \simeq 10^{-30} \mathrm{~cm}^{3} \mathrm{~s}^{-1}$ and $r=0.07$ are considered.

Fig. 2 The magnetic like linear polarization angular power spectrum $l(l+1) /(2 \pi) C_{l}^{B B}$ in units of $(\mu K)^{2}$ is plotted for different values of the tensor to scalar ratio $r$; thick black, thick gray, dashed-dotted red, dashed blue, dashed pink, dotted green and dashed-dotted brown lines indicate $C_{l}^{B B}$ due to: the gravitation lensing effects, the standard contribution due to Compton scattering in the presence of tensor per-

where $\bar{C}_{l}^{\mathrm{EE}, \mathrm{S}}$ is the value of the power spectrum for E mode polarization associated with the Compton scattering in the contex of scalar perturbations $[34,35]$

$\bar{C}_{l}^{\mathrm{EE}, \mathrm{S}}=(4 \pi)^{2} \frac{(l+2) !}{(l-2) !} \int d^{3} K P_{\mathrm{S}}(K)\left|\frac{3}{4} \int_{0}^{\eta_{0}} d \eta g_{\mathrm{e}}(\eta) \Pi(\eta, K) \frac{j_{l}}{x^{2}}\right|^{2}$,

and $\bar{\tau}_{\mathrm{DM}}$ is the time average of $\tau_{\mathrm{DM}}$

$$
\begin{aligned}
\bar{\tau}_{\mathrm{DM}}(z)= & \frac{1}{z_{l}} \int_{0}^{z_{l}} d z \tau_{\mathrm{DM}}(z) \approx \frac{1.4 \times 10^{-1} \mathrm{MeV}^{2}}{m_{\mathrm{DM}}^{2}} \\
& \times\left(\frac{\langle\sigma v\rangle}{10^{-30 \frac{\mathrm{cm}^{3}}{\mathrm{~s}}}}\right)^{1 / 2}\left(\frac{\rho_{\mathrm{DM}}^{0}}{2.5 \times 10^{-30} \frac{\mathrm{g}}{\mathrm{cm}^{3}}}\right),
\end{aligned}
$$

with $z_{l} \approx 1100$ represents redshift at the last scattering surface. Therefore, one can expect a valuable contribution on the $C_{l}^{\mathrm{BB}, \mathrm{S}}$ from DM particles with masses about a few $\mathrm{MeV}$.

\section{Conclusion and discussion}

We have considered the scattering of the CMB-photons from DDM. Consequently, producing the magnetic-like linear polarization power spectrum of the CMB photons has been estimated by using quantum Boltzmann equation. To this end, the $\mathrm{CMB}$ radiation has been considered as an ensemble of photons which is described by the Stokes parameters. Meanwhile, the Compton scattering and the photon-DDM interaction have been considered as the collision terms in

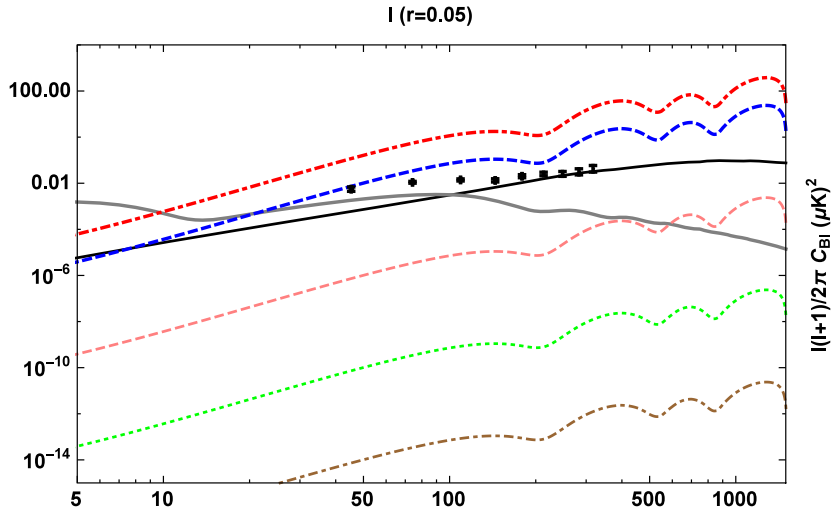

(b) To plot above curves, $\langle\sigma v\rangle \simeq 10^{-30} \mathrm{~cm}^{3} \mathrm{~s}^{-1}$ and $r=0.05$ are considered.

turbations with $r$ mentioned in sub-caption, the DM magnetic moment contribution in the presence of scalar metric perturbations with different masses $m_{D} \equiv\{1 \mathrm{MeV} ; 5 \mathrm{MeV} ; 100 \mathrm{MeV} ; 500 \mathrm{MeV} ; 1 \mathrm{GeV}\}$, respectively. The points with error bars show the BICEP2/Keck Array data. We have chosen the Planck best fit values for the cosmological parameters

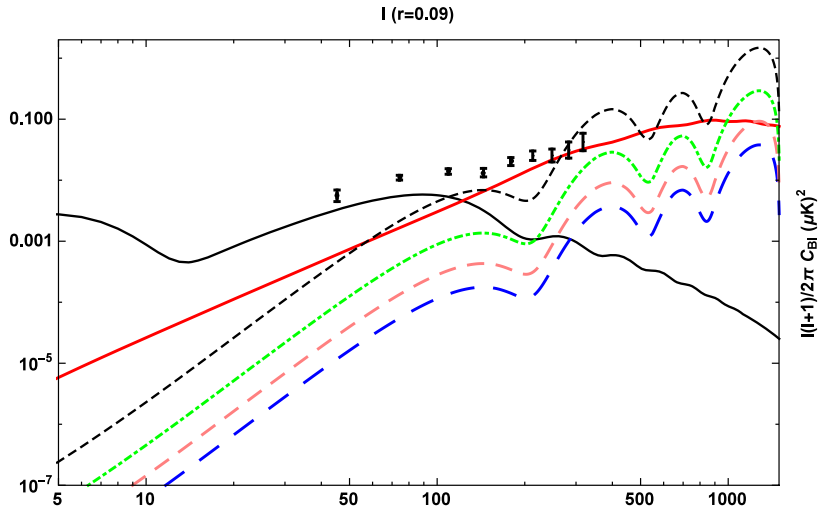

(a) To plot above curves, $\langle\sigma v\rangle \simeq 10^{-30} \mathrm{~cm}^{3} \mathrm{~s}^{-1}$ and

$$
r=0.09 \text { are considered. }
$$

Fig. 3 The magnetic like linear polarization angular power spectrum $l(l+1) /(2 \pi) C_{l}^{B B}$ in units of $(\mu K)^{2}$ is plotted; thick red, thick black, dashed black, dotted green, dashed pink, dashed blue lines indicate $C_{l}^{B B}$ due to: the gravitation lensing effects, the standard contribution due to Compton scattering in the presence of tensor perturbations, the DM magnetic moment contribution in the presence of scalar metric perturbations with different masses $m_{D} \equiv$ $\{70 \mathrm{MeV}, 80 \mathrm{MeV}, 90 \mathrm{MeV}, 100 \mathrm{MeV}\}$, respectively. The points with error bars show the BICEP2/Keck Array data. We have chosen the Planck best fit values for the cosmological parameters

the quantum Boltzmann equation. For the DM we have considered both Dirac and Majorana particles as candidates for the DDM. We have shown that only the Majorana particles through a transition magnetic moment with one chirality or two chiralities with different couplings can produce the CMB polarization. Nevertheless, we have considered the 


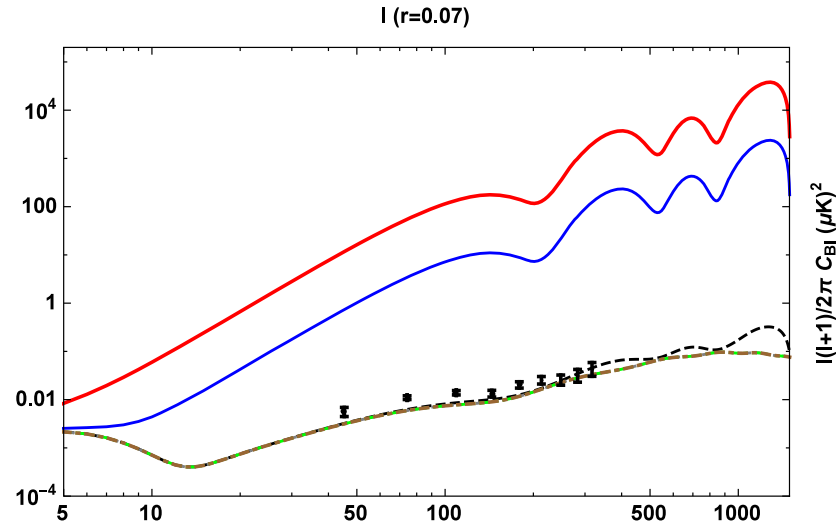

(a) To plot above curves, $\langle\sigma v\rangle \simeq 10^{-30} \mathrm{~cm}^{3} \mathrm{~s}^{-1}$ and

$$
r=0.07 \text { are considered. }
$$

Fig. 4 The magnetic like linear polarization angular power spectrum $l(l+1) /(2 \pi) C_{l}^{B B}$ in units of $(\mu K)^{2}$ is plotted for different values of the tensor to scalar ratio $r$ and $\langle\sigma v\rangle$; the diagrams show: Compton scattering in the presence of tensor perturbations and gravitation lensing effect without considering DDM interactions (thick-dashed black line), Compton scattering in the presence of tensor perturbations and gravitation lensing plus DDM interactions in the presence of scalar

right handed Majorana DM to explore the power spectrum of the B-mode polarization of the CMB photons. The most important point is that the B-mode polarization pattern has been generated by the CMB-DDM interaction in the presence of the scalar perturbation. This is while the standard scenario for the generation of the CMB B-mode polarization assumes that this type of polarization pattern can only be produced due to the Compton scattering in the presence of tensor perturbations. However, to obtain the numerical results and to find some bounds on the magnetic dipole moment two cases have been taken into account. In the first case, the Majorana particle mass splitting is smaller than the energy of the CMB photons that leads to suppression for decaying of the heavier DM for the magnetic moment as large as $10^{-16} e \mathrm{~cm}$, see (63). In contrast, in the second case where the mass splitting of the two Majorana particles is larger than the energy of the CMB photons, the decay process can occur but the CMB polarization is suppressed as $\left(\frac{k^{0}}{\delta}\right)^{2}$ as is shown in (59). In fact, only for the case one where the DM particles are stable a valuable polarization can be produced.

To compare the contribution of the CMB-DDM interaction in the case one where $m_{\mathrm{DM}_{1}} \sim m_{\mathrm{DM}_{2}}=m_{\mathrm{DM}}$ with respect to the Compton scattering, the numerical value of the $\mathrm{B}$-mode power spectrum of the $\mathrm{CMB}$ has been plotted for different values of $m_{\mathrm{DM}}$ and $r$ and for $\langle\sigma v\rangle \approx 10^{-30} \mathrm{~cm}^{3} \mathrm{~s}^{-1}$ together with BICEP2/Keck Array data (see Figs. 2, 3, 4 and 5). Figures 2 and 3 show the behavior of the B-mode power spectrum due to the Compton scattering in the presence of tensor perturbations, the gravitational lensing effects and photon-DDM interaction in the case of scalar perturba-
I ( $r=0.05)$

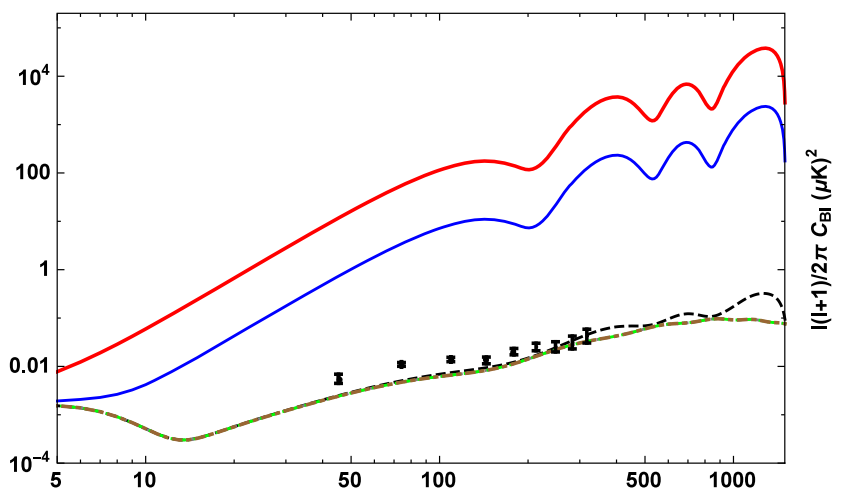

(b) To plot above curves, $\langle\sigma v\rangle \simeq 10^{-30} \mathrm{~cm}^{3} \mathrm{~s}^{-1}$ and $r=0.05$ are considered.

perturbations by considering different masses for DM $m_{D}=1 \mathrm{MeV}$ (red line), $m_{D}=5 \mathrm{MeV}$ (blue line), $m_{D}=100 \mathrm{MeV}$ (dashed Pink line), $m_{D}=500 \mathrm{MeV}$ (dotted geen line) and $m_{D}=1 \mathrm{GeV}$ (dasheddotted brown line) respectively. The points with error bars show the BICEP2/Keck Array data. We have chosen the Planck best fit values for the cosmological parameters

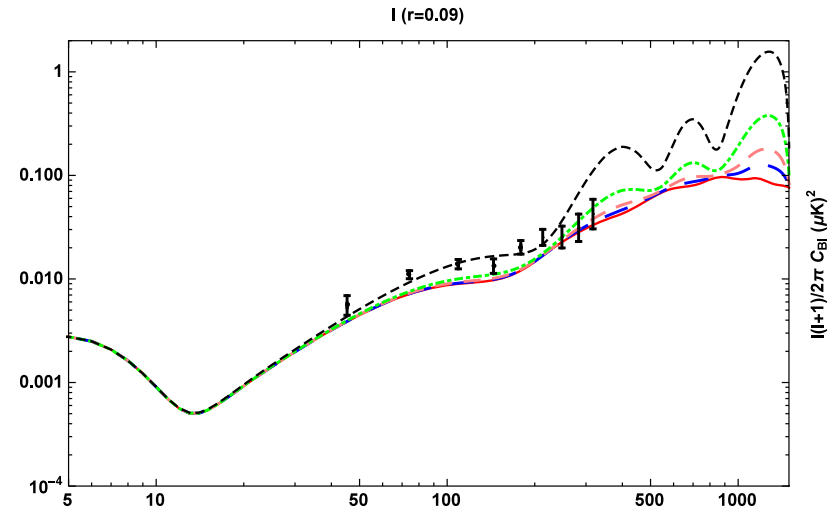

(a) To plot above curves, $\langle\sigma v\rangle \simeq 10^{-30} \mathrm{~cm}^{3} \mathrm{~s}^{-1}$ and

$$
r=0.09 \text { are considered. }
$$

Fig. 5 The magnetic like linear polarization angular power spectrum $l(l+1) /(2 \pi) C_{l}^{B B}$ in units of $(\mu K)^{2}$ is plotted; The plot shows: Compton scattering in the presence of tensor perturbations with $r=0.09$ and gravitation lensing effect without considering DDM interactions (red line), Compton scattering in the presence of tensor perturbations and gravitation lensing plus DDM interactions in the presence of scalar perturbations by considering different masses for DM $m_{D}=70 \mathrm{MeV}$ (black line), $m_{D}=80 \mathrm{MeV}$ (green line), $m_{D}=90 \mathrm{MeV}$ (dashed pink line), $m_{D}=100 \mathrm{MeV}$ (dashed blue line), respectively. The points with error bars show the BICEP2/Keck Array data. We have chosen the Planck best fit values for the cosmological parameters

tion. On the other hand, Figs. 4 and 5 indicate the contribution of the lensing effects and the Compton scattering in the presence of tensor perturbation plus photon-DDM interaction. As the figures show for different values of the $r$ parameter one can obtain different mass regions for DDM. For example, 
the contribution of the B-mode due to CMB-DDM interaction in the presence of scalar perturbations for $r=0.07$ and $m_{\mathrm{DM}} \leq 50 \mathrm{MeV}\left(r=0.09\right.$ and $\left.m_{\mathrm{DM}} \leq 80 \mathrm{MeV}\right)$ is larger than the total reported B-mode power spectrum and therefore can be excluded experimentally. In fact, the B-mode polarization power spectrum can put a bound on the magnetic dipole moment about $M \leq 10^{-16} e \mathrm{~cm}$ which is in agreement with other reported constraints [12,5254].

It should be emphasized that the $r$ ratio is usually introduced by comparing B- and E-modes linear polarization power spectrum while it is assumed that the observed B-mode $C_{\mathrm{Bl}}^{\mathrm{ob}}$ is totally attributed to the Compton scattering in the presence of tensor perturbations $C_{\mathrm{Bl}}^{\mathrm{ob}}=C_{\mathrm{Bl}}^{T}$. However, our results show that other interactions such as CMB-DDM interaction can generate magnetic like power spectrum in the presence of scalar perturbations $C_{\mathrm{Bl}}^{(\mathrm{S})}$ and therefore $C_{\mathrm{Bl}}^{\mathrm{ob}}=C_{\mathrm{Bl}}^{T}+C_{\mathrm{Bl}}^{(\mathrm{S})}$. So, the $r$-parameter is modified as follows

$\mathrm{r} \propto \mathrm{C}_{\mathrm{Bl}}^{\mathrm{T}} / \mathrm{C}_{\mathrm{El}}^{(\mathrm{S})} \propto\left(\mathrm{C}_{\mathrm{Bl}}^{\mathrm{ob}}-\mathrm{C}_{\mathrm{Bl}}^{(\mathrm{S})}\right) / \mathrm{C}_{\mathrm{El}}^{(\mathrm{S})}$.

As (91) shows, the value of the $r$-parameter, as a scale of the amplitude of gravitational wave, is suppressed. By using (81-90) in (91), one can approximately find

$r \simeq r^{0}-\sin ^{2} \bar{\tau}_{\mathrm{DM}}$

where $r^{0}$ is the standard tensor to scalar perturbation ratio without considering any new source for the B-mode polarization such as the CMB-DDM interaction.

As a final point, what we did not calculate in this paper are the resonance mode $\delta \sim k^{0}$ and the contribution of electric dipole moment for the Majorana particles which is under investigation as a future work [55]. However, we should mention that the future observed data for the B-mode polarization power spectrum can be used as an indirect probe of the nature of photon-DM interaction.

Data Availability Statement This manuscript has no associated data or the data will not be deposited. [Authors' comment: In this article, we have entered our data as much as possible. However, if anyone has any questions or comments, we are happy to discuss and learn.]

Open Access This article is licensed under a Creative Commons Attribution 4.0 International License, which permits use, sharing, adaptation, distribution and reproduction in any medium or format, as long as you give appropriate credit to the original author(s) and the source, provide a link to the Creative Commons licence, and indicate if changes were made. The images or other third party material in this article are included in the article's Creative Commons licence, unless indicated otherwise in a credit line to the material. If material is not included in the article's Creative Commons licence and your intended use is not permitted by statutory regulation or exceeds the permitted use, you will need to obtain permission directly from the copyright holder. To view a copy of this licence, visit http://creativecomm ons.org/licenses/by/4.0/.

Funded by SCOAP ${ }^{3}$.

\section{Appendix A: Forward scattering of photon-majorana DM with both chiralities}

In this Appendix, we are going to calculate the contribution of the photon-Majorana DDM forward scattering in detail.

Based on the Lagrangian which has been introduced in (33), two Majorana fermions $\chi_{1}$ and $\chi_{2}$, with mass splitting $\delta$, can interact with photons through transition magnetic moment. The Feynman diagram corresponding to the lowest order interaction has been shown in Fig. 1. By using Lagrangian (33) and Fig. 1, the total Feynman amplitude is given by

$$
\begin{aligned}
\mathcal{M}= & M_{12}^{2}\left(\frac{\bar{u}_{r^{\prime}}\left(q^{\prime}\right) \notin_{s^{\prime}}\left(p^{\prime}\right) \not p^{\prime}\left(q+\not p+m_{\left.\mathrm{DM}_{2}\right) \notin_{s}(p) \not p} u_{r}(q)\right.}{(p+q)^{2}-m_{\mathrm{DM}_{2}}^{2}}\right. \\
& +\frac{\bar{u}_{r^{\prime}}\left(q^{\prime}\right) \notin_{s}(p) \not p\left(q-\not p^{\prime}+m_{\left.\mathrm{DM}_{2}\right) \notin_{s^{\prime}}}\left(p^{\prime}\right) \not p^{\prime} u_{r}(q)\right.}{\left(-q+p^{\prime}\right)^{2}-m_{\mathrm{DM}_{2}}^{2}} \\
& +\frac{\bar{v}_{r}(q) \notin_{s}(p) \not p\left(-q-\not p+m_{\left.\mathrm{DM}_{2}\right) \notin_{s^{\prime}}\left(p^{\prime}\right) \not p^{\prime}} v_{r^{\prime}}\left(q^{\prime}\right)\right.}{(p-q)^{2}-m_{\mathrm{DM}_{2}}^{2}} \\
& \left.+\frac{\bar{v}_{r}(q) \notin_{s^{\prime}}\left(p^{\prime}\right) \not p^{\prime}\left(-q+\not p^{\prime}+m_{\mathrm{DM}_{2}}\right) \notin_{s}(p) \not p v_{r^{\prime}}\left(q^{\prime}\right)}{\left(-q+p^{\prime}\right)^{2}-m_{\mathrm{DM}_{2}}^{2}}\right)+(1 \leftrightarrow 2) .
\end{aligned}
$$

According to (37), to calculate the forward scattering term, we should impose the conditions $q^{\prime}=q$ and $p^{\prime}=p=k$ to the Feynman amplitude which leads to

$$
\begin{aligned}
\mathcal{M}= & \frac{M_{12}^{2}}{(2 k \cdot q)^{2}-\left(m_{\mathrm{DM}_{2}}^{2}-m_{\mathrm{DM}_{1}}^{2}\right)^{2}} \\
& \times\left(\left(2 k \cdot q+\left(m_{\mathrm{DM}_{2}}^{2}-m_{\mathrm{DM}_{1}}^{2}\right)\right)(2 k \cdot q)\right. \\
& \times \bar{u}_{r}(q) \notin_{s^{\prime}}(k) \notin_{s}(k) \not k \quad u_{r}(q) \\
& -\left(2 k \cdot q-\left(m_{\mathrm{DM}_{2}}^{2}-m_{\mathrm{DM}_{1}}^{2}\right)\right)(2 k \cdot q) \\
& \times \bar{u}_{r}(q) \notin_{s}(k) \notin_{s^{\prime}}(k) \not k \quad u_{r}(q) \\
& +\left(2 k \cdot q+\left(m_{\mathrm{DM}_{2}}^{2}-m_{\mathrm{DM}_{1}}^{2}\right)\right)(2 k \cdot q) \\
& \times \bar{v}_{r}(q) \notin_{s}(k) \notin_{s^{\prime}}(k) \not k \quad v_{r}(q) \\
& -\left(2 k \cdot q-\left(m_{\mathrm{DM}_{2}}^{2}-m_{\mathrm{DM}_{1}}^{2}\right)\right) \\
& \left.(2 k \cdot q) \bar{v}_{r}(q) \notin_{s^{\prime}}(k) \notin_{s}(k) k v_{r}(q)\right)+(1 \leftrightarrow 2)
\end{aligned}
$$

or

$$
\begin{aligned}
\mathcal{M}= & \frac{M_{12}^{2}}{(2 k \cdot q)^{2}-\left(m_{\mathrm{DM}_{2}}^{2}-m_{\mathrm{DM}_{1}}^{2}\right)^{2}} \\
& \times(2 k \cdot q)^{2}\left(\bar{u}_{r}(q)\left(\xi_{s^{\prime}}(k) \notin_{s}(k)-\xi_{s}(k) \notin_{s^{\prime}}(k)\right) \not k u_{r}(q)\right. \\
& \left.+\bar{v}_{r}(q)\left(\xi_{s}(k) \notin_{s^{\prime}}(k)-\xi_{s^{\prime}}(k) \xi_{s}(k)\right) \not k v_{r}(q)\right)
\end{aligned}
$$




$$
\begin{aligned}
& +2 k \cdot q\left(m_{\mathrm{DM}_{2}}^{2}-m_{\mathrm{DM}_{1}}^{2}\right) \\
& \times\left(\bar{u}_{r}(q)\left(\notin_{s^{\prime}}(k) \notin_{s}(k)+\notin_{s}(k) \notin_{s^{\prime}}(k)\right) \not k u_{r}(q)\right. \\
& \left.+\bar{v}_{r}(q)\left(\notin_{s}(k) \notin_{s^{\prime}}(k)+\notin_{s^{\prime}}(k) \notin_{s}(k)\right) \not k v_{r}(q)\right)+(1 \leftrightarrow 2) .
\end{aligned}
$$

Since the terms proportional to the mass difference lead to the product of polarization vectors, $\epsilon_{s} \cdot \epsilon_{s^{\prime}}=-\delta_{s s^{\prime}}$, their contributions will be zero. Using the relations (30) and (31) one can find that the contributions of the remaining terms lead to zero and therefore this interaction can not affect the CMB polarization.

\section{References}

1. F. Zwicky, Helv. Phys. Acta 6, 110 (1933)

2. S. Smith, Astrophys. J. 83, 23 (1936)

3. V.C. Rubin, W.K. Ford, Astrophys. J. 159, 379 (1970)

4. S.M. Faber, J.S. Gallagher, Annu. Rev. Astron. Astrophys. 17, 135 (1979)

5. V.C. Rubin, W.K. Ford, N. Thonnard, Astrophys. J. 238, 471 (1980)

6. K.G. Begeman, A.H. Broeils, R.H. Sanders, Mon. Not. R. Astron. Soc. 249, 523 (1991)

7. R.P. Olling, M.R. Merrifield, Mon. Not. R. Astron. Soc. 311, 361 (2000)

8. M. Fich, S. Tremaine, Annu. Rev. Astron. Astrophys. 29, 409 (1991)

9. R. Bernabei et al. [DAMA and LIBRA Collaborations], Eur. Phys. J. C 67, 39 (2010). https://doi.org/10.1140/epjc/ s10052-010-1303-9. arXiv:1002.1028 [astro-ph.GA]

10. C.E. Aalseth, P.S. Barbeau, N.S. Bowden et al., Phys. Rev. Lett. 106, 131301 (2011)

11. R. Agnese et al. [CDMS Collaboration], Phys. Rev. D 88, 031104 (2013). https://doi.org/10.1103/PhysRevD.88.031104, https://doi.org/10.1103/PhysRevD.88.059901. arXiv:1304.3706 [astro-ph.CO] [Erratum: Phys. Rev. D 88, no. 5, 059901 (2013)]

12. K. Sigurdson, M. Doran, A. Kurylov, R.R. Caldwell, M. Kamionkowski, Phys. Rev. D 70, 083501 (2004). https:// doi.org/10.1103/PhysRevD.70.083501, https://doi.org/10.1103/ PhysRevD.73.089903. arXiv:astro-ph/0406355 [Erratum: Phys. Rev. D 73, 089903 (2006)]

13. E. Masso, S. Mohanty, S. Rao, Phys. Rev. D 80, 036009 (2009). https://doi.org/10.1103/PhysRevD.80.036009. arXiv:0906.1979 [hep-ph]

14. S. Gardner, Phys. Rev. D 79, 055007 (2009)

15. J. Bagnasco, M. Dine, S.D. Thomas, Phys. Lett. B 320, 99 (1994)

16. W.S. Cho, J.H. Huh, I.W. Kim, J.E. Kim, B. Kyae, Phys. Lett. B 687, 6 (2010) [Phys. Lett. B 694, 496 (2011)]

17. V. Barger, W.Y. Keung, D. Marfatia, Phys. Lett. B 696, 74 (2011). https://doi.org/10.1016/j.physletb.2010.12.008. arXiv:1007.4345 [hep-ph]

18. H. An, S.-L. Chen, R.N. Mohapatra, S. Nussinov, Y. Zhang, Phys. Rev. D 82, 23533 (2010)

19. M. Giovannini, K.E. Kunze, Phys. Rev. D 78, 023010 (2008). arXiv:0804.3380 [astro-ph]

20. E. Bavarsad, M. Haghighat, Z. Rezaei, R. Mohammadi, I. Motie, M. Zarei, Phys. Rev. D 81, 084035 (2010). https://doi.org/10.1103/ PhysRevD.81.084035. arXiv:0912.2993 [hep-th]

21. R. Mohammadi, Eur. Phys. J. C 74(10), 3102 (2014). https://doi. org/10.1140/epjc/s10052-014-3102-1, https://doi.org/10.1140/ epjc/s10052-014-3089-7. arXiv:1312.2199 [astro-ph.CO]
22. R. Mohammadi, S.S. Xue, Phys. Lett. B 731, 272 (2014). https:// doi.org/10.1016/j.physletb.2014.02.031. arXiv:1312.3862 [hep$\mathrm{ph}]$

23. I. Motie, S.S. Xue, EPL 100(1), 17006 (2012). https://doi.org/10. 1209/0295-5075/100/17006. arXiv:1104.3555 [hep-ph]

24. S. Shakeri, M. Haghighat, S.S. Xue, JCAP 1710, 014 (2017). $\quad$ https://doi.org/10.1088/1475-7516/2017/10/014. arXiv:1704.04750 [astro-ph.HE]

25. S. Batebi, R. Mohammadi, R. Ruffini, S. Tizchang, S.S. Xue, Phys. Rev. D 94(6), 065033 (2016). https://doi.org/10.1103/PhysRevD. 94.065033. arXiv:1610.01154 [astro-ph.HE]

26. P. Montero-Camacho, C.M. Hirata, JCAP 1808, 040 (2018). https:// doi.org/10.1088/1475-7516/2018/08/040. arXiv:1803.04505 [astro-ph.CO]

27. M. Kamionkowski, Phys. Rev. D 97(12), 123529 (2018). https:// doi.org/10.1103/PhysRevD.97.123529. arXiv:1804.06412 [astroph.CO]

28. S. Modares Vamegh, M. Haghighat, S. Mahmoudi, R. Mohammadi, Phys. Rev. D 100(10) (2019). https://doi.org/10.1103/PhysRevD. 100.103024. arXiv: 1911.02264 [hep-ph]

29. M. Haghighat, S. Mahmoudi, R. Mohammadi, S. Tizchang, S.S. Xue, (2019). arXiv:1909.03883 [hep-ph]

30. A. Vahedi, J. Khodagholizadeh, R. Mohammadi, M. Sadegh, JCAP 1901, 052 (2019). https://doi.org/10.1088/1475-7516/2019/ 01/052. arXiv:1809.08137 [astro-ph.CO]

31. A. Kosowsky, Ann. Phys. 246, 49 (1996). https://doi.org/10.1006/ aphy.1996.0020. arXiv:astro-ph/9501045

32. M. Kamionkowski, A. Kosowsky, A. Stebbins, Phys. Rev. D 55, 7368 (1997). https://doi.org/10.1103/PhysRevD.55.7368. arXiv:astro-ph/9611125

33. W. Hu, M.J. White, Phys. Rev. D 56, 596 (1997). https://doi.org/ 10.1103/PhysRevD.56.596. arXiv:astro-ph/9702170

34. M. Zaldarriaga, U. Seljak, Phys. Rev. D 55, 1830 (1997). https:// doi.org/10.1103/PhysRevD.55.1830. arXiv:astro-ph/9609170

35. U. Seljak, M. Zaldarriaga, Astrophys. J. 469, 437 (1996). https:// doi.org/10.1086/177793. arXiv:astro-ph/9603033

36. C. Scoccola, D. Harari, S. Mollerach, Phys. Rev. D 70, 063003 (2004). https://doi.org/10.1103/PhysRevD.70.063003. arXiv:astro-ph/0405396

37. J. Khodagholizadeh, R. Mohammadi, S.S. Xue, Phys. Rev. D 90(9), 091301 (2014). https://doi.org/10.1103/PhysRevD.90. 091301. arXiv:1406.6213 [astro-ph.CO]

38. R. Mohammadi, J. Khodagholizadeh, M. Sadegh, S.S. Xue, Phys. Rev. D 93(12), 125029 (2016). https://doi.org/10.1103/PhysRevD. 93.125029. arXiv:1602.00237 [astro-ph.CO]

39. S. Tizchang, S. Batebi, M. Haghighat, R. Mohammadi, Eur. Phys. J. C 76(9), 478 (2016). https://doi.org/10.1140/epjc/ s10052-016-4312-5. arXiv:1605.09045 [hep-ph]

40. J. Khodagholizadeh, R. Mohammadi, M. Sadegh, A. Vahedi, (2019). arXiv:1909.00568 [astro-ph.CO]

41. P.A.R. Ade et al. [BICEP2 and Planck Collaborations], Phys. Rev. Lett. 114, 101301 (2015). https://doi.org/10.1103/PhysRevLett. 114.101301. arXiv:1502.00612 [astro-ph.CO]

42. M.M. Ettefaghi, M. Haghighat, Phys. Rev. D 77, 056009 (2008). https://doi.org/10.1103/PhysRevD.77.056009. arXiv:0712.4034 [hep-ph]

43. H.M. Lee, Phys. Lett. B 738, 118 (2014). https://doi.org/10.1016/ j.physletb.2014.09.031. arXiv:1404.5446 [hep-ph]

44. P.W. Angel, N.L. Rodd, R.R. Volkas, Phys. Rev. D 87(7), 073007 (2013). https://doi.org/10.1103/PhysRevD.87.073007. arXiv:1212.6111 [hep-ph]

45. A. Aparici, K. Kim, A. Santamaria, J. Wudka, Phys. Rev. D 80, 013010 (2009). https://doi.org/10.1103/PhysRevD.80.013010. arXiv:0904.3244 [hep-ph] 
46. O. Antipin, M. Redi, A. Strumia, E. Vigiani, JHEP 1507, 039 (2015). https://doi.org/10.1007/JHEP07(2015)039. arXiv:1503.08749 [hep-ph]

47. X. Chu, J. Pradler, L. Semmelrock, Phys. Rev. D 99(1), 015040 (2019). https://doi.org/10.1103/PhysRevD.99.015040. arXiv:1811.04095 [hep-ph]

48. C. Itzykson, J.B. Zuber, Quantum Field Theory (Mcgraw-Hill, New York, 1980). (International Series In Pure and Applied Physics)

49. S. Davidson, M. Gorbahn, A. Santamaria, Phys. Lett. B 626, 151 (2005). https://doi.org/10.1016/j.physletb.2005.08.086. arXiv:hep-ph/0506085

50. M. Paraskevas, (2018). arXiv:1802.02657 [hep-ph]
51. P.A.R. Ade et al. [Planck Collaboration], Astron. Astrophys. 594, A13 (2016). https://doi.org/10.1051/0004-6361/201525830. arXiv:1502.01589 [astro-ph.CO]

52. I. Lopes, K. Kadota, J. Silk, Astrophys. J. Lett. 780, L15 (2014). https://doi.org/10.1088/2041-8205/780/2/L15. arXiv:1310.0673 [astro-ph.SR]

53. E. Del Nobile, F. Sannino, IJMPA 27, 50065 (2012)

54. J.-F. Fortin, T.M.P. Tait, Phys. Rev. D 85, 63506 (2012)

55. S. Mahmoudi, M. Haghighat, R. Mohammadi, S. Modares Vamegh, (2020) (in preparation) 